\title{
Development of quality index to classify meal healthiness through photos: first step for app of meal assessment using Machine Learning
}

\author{
Josiane Steluti ${ }^{1}$, Jun Okamoto Junior ${ }^{2}$ and Dirce Maria Lobo Marchioni ${ }^{1}$ \\ ${ }^{1}$ School of Public Health, University of São Paulo, São Paulo, Brazil and \\ ${ }^{2}$ Escola Politécnica, University of São Paulo, São Paulo, Brazil
}

\begin{abstract}
Introduction: Currently, there are approximately 84 million smartphones in use in Brazil. The use of this technology facilitates the daily life of the individuals in the personal, social and professional scope. Therefore, we hypothesize that it can be used to assess and improve dietary intake. Thus, we intend to develop an application to assess meal quality from a photo taken by a smartphone, using artificial intelligence. For this, the machine should be trained to recognize which meal is healthy or unhealthy, and, as a first step, a meal quality index is necessary.
\end{abstract}

Objective: This study aims to develop a meal quality index, to be applied to photos of dishes from a main meal.

Methods: For the development of the index, it was considered the main recommendations established in the scientific literature and the "Dietary Guidelines for the Brazilian Population". The index includes nine components: 1- meat intake and/or meat substitute, 2cooking method, 3- vegetables intake, 4- whole food intake, 5- food variety, 6- ultra-processed food intake, 7- fruits intake, 8- carbohydrates intake, 9- fat recipes and/or food. First, questions were elaborated and scored as 0 point (unhealthy answer) or 1 point (healthy answer). After, the meal photo was classified as "needs improvement" ( $<4$ points), good ( $>5 \mathrm{e}<7$ points) and very good ( $>8$ points). Each photo was assessed by two experts. Then, statistical analyses were performed considering Kappa (k) statistic to evaluate the agreement between the assessments by experts.

Results: Data from 154 meal photos were assessed. We analyzed the \% of agreement, k-value and significant agreement ( $p$-value) for all index components and final classification, respectively: 1- meat intake and/or meat substitute, $64.94 \%$ and $\mathrm{k}=0.2759(p<0.001) ; 2$ cooking method, $81.82 \%$ and $\mathrm{k}=0.5915(p=0.000) ; 3$ - vegetables intake, $77.27 \%$ and $\mathrm{k}=0.5353(p=0.000)$; 4- whole food intake, $98.05 \%$ and $\mathrm{k}=-0.0087(p=0.545) ; 5$ - food variety, $79.22 \%$ and $\mathrm{k}=0.5899(p=0.000) ; 6$ - ultra processed food intake, $76.62 \%$ and $\mathrm{k}=0.4515(p=0.000) ; 7$ - fruits intake, $94.81 \%$ and $\mathrm{k}=0.8494(p=0.000) ; 8$ - carbohydrates intake, $65.58 \%$ and $\mathrm{k}=0.2960(p=$ $0.000)$; 9- fat recipes and/or food, $73.38 \%$ and $\mathrm{k}=0.4654(p=0.000)$; and final classification, $58.44 \%$ and $\mathrm{k}=0.3218(p=0.000)$.

Conclusion: We verified a moderate and significant agreement for almost all index components using meal photo.

\section{Conflict of Interest}

There is no conflict of interest. 